\title{
Clinical Profile and Laboratory Findings of Dengue Fever in Children
}

\author{
Mahesh Ahirrao', Ashish Patil ${ }^{2}$, Anjali Kurup ${ }^{3}$, Aditya Thorat ${ }^{4}$, \\ Komal Shivnani ${ }^{5}$, Suriya Patil ${ }^{6}$ \\ ${ }^{1}$ Professor, Department of Pediatrics, ACPM Medical College, Dhule \\ ${ }^{2}$ Junior Resident 3, Department of Pediatrics, ACPM Medical College, Dhule \\ ${ }^{3}$ Junior Resident 3, Department of Pediatrics, ACPM Medical College, Dhule \\ ${ }^{4}$ Junior Resident 2, Department of Pediatrics, ACPM Medical College, Dhule \\ ${ }^{5}$ Junior Resident 2, Department of Pediatrics, ACPM Medical College, Dhule \\ ${ }^{6}$ Junior Resident 2, Department of Pathology, PDMMC, Amravati \\ Corresponding Author: Ashish Patil
}

\begin{abstract}
Background: Dengue is an arthropod borne viral hemorrhagic fever. It is a major public health concern throughout the tropical and subtropical regions of the world.

Objectives: To study clinical profile and laboratory findings of dengue infection in children.

Materials and Methods: Children up to the age of 15 years who were confirmed serologically dengue positive (either positive NS1 antigen or positive IgM antibody by ELISA method were included in study. Total 100 patients were studied. Pre tested, semi structured questionnaire were developed and used for data collection.

Result and Discussion: In current study, maximum patients were found in Dengue Hemorrhagic Fever (DHF) group (68\%), followed by Dengue Fever (25\%). Fever was most common clinical manifestation. Maximum patients in this study were in 10 to 15 years of age group, male being more commonly affected. Thrombocytopenia and leukopenia were present in $90 \%$ and $69 \%$ of patients respectively.

Conclusion: School going children were more exposed to mosquito bites during day time school activities, thus maximum patients were from 10 to 15 years of age group. In early diagnosis of dengue fever, ELISA NS1 antigen is seen more promisable. When used in combination with ELISA IgM antibody, it significantly improves the diagnostic algorithm. Thrombocytopenia is more consistent feature of Dengue Hemorrhagic Fever (DHF) and Dengue Shock Syndrome (DSS).
\end{abstract}

Keywords: Dengue Hemorrhagic Fever (DHF), Dengue Shock Syndrome (DSS), ELISA NS1 antigen, ELISA IgM antibody

\section{INTRODUCTION}

Dengue is the most rapidly spreading mosquito-borne viral disease of mankind, with a 30-fold increase in global incidence over the last three decades ${ }^{1}$. Dengue fever is an acute febrile infectious disease in subtropical and tropical areas that is progressively making its way from being "one of the great neglected diseases of mankind" towards being acknowledged as one of the world's major infectious disease $^{2,3}$. Dengue is a fast emerging and rapidly spreading systemic viral infection with global estimates of 390 million infections per year, of which 96 million are apparent infections and 3.97 billion people in 128 countries are at risk of dengue infection ${ }^{4}$. Dengue is one of the most common vector-borne diseases in Southeast Asia and has been ranked as the most important mosquito-borne viral disease with epidemic potential in the world ${ }^{5}$. Some 2.5 
billion people - two fifths of the world's population in tropical and subtropical countries are at risk ${ }^{6}$. The etiologic agents include all four serotypes which belong to the genus Flavivirus in the family Flaviviridae $^{7}$. Four dengue serotypes, known as DENV-1, DENV-2, DENV-3, and DENV-4, are genetically related and biological similar, can cause severe and fatal disease ${ }^{8}$. The principle vector is female mosquito, Aedes aegypti, which breeds largely indoors in clean waters mainly in artificial water containers, and feeds on humans in daytime ${ }^{9}$. The clinical manifestations of dengue vary with the age and immunity of the patient. It can present as 1) an inapparent infection 2) nonspecific febrile illness, 3) classic dengue fever, 4) dengue hemorrhagic fever, 5) Dengue shock syndrome (DSS) and 6) encephalopathy and 7) fulminant liver Failure $^{10}$.

After incubation, the disease begins abruptly and \& clinical course of illness passes through the following three phases:

1) Febrile phase

2) Critical phase

3) Convalescent phase $^{11,12}$. The present study was carried out to study clinical profile and laboratory findings, of dengue infection in city and rural area.

\section{AIMS AND OBJECTIVES}

- To study the clinical profile of patients with dengue fever.

- To study the various laboratory parameters in patients with dengue fever.

\section{MATERIALS AND METHODOLOGY STUDY DESIGN:-}

A prospective observational study.

\section{STUDY DURATION:-}

Study Duration was 18 months, from 1st January 2018 till 30th June 2019.

\section{STUDY POPULATION:-}

The present study was conducted on children up to 15 years of age who were confirmed serological dengue positive (either Positive NS1 antigen or Positive Ig $\mathrm{M}$ antibody by ELISA method), referred to tertiary care hospital.

\section{SAMPLE SIZE:-}

Sample size was 100 children up to 15 years of age who fulfilled the inclusion criteria.

\section{SAMPLING TECHNIQUE (SAMPLING METHOD):-} was used.

Simple random sampling technique

\section{SELECTION CRITERIA:- INCLUSION CRITERIA:-}

1. All patients between the age group of $0-$ 15 years.

2. Patients with confirmed diagnosis of dengue fever (positive test for NS1 antigen/IgM antibody by ELISA method).

\section{EXCLUSION CRITERIA:-}

1. Patient with serological test negative for dengue.

\section{METHODOLOGY OF STUDY:}

The pre-tested, semi structured questionnaire developed and used for data collection. Patient / Patient guardian/ Patient relative were informed and explained about the study. Written informed consent had been taken prior to procedures as per attached proforma.

- Socioeconomic data was collected.

- Clinical histories were noted down along with thorough clinical examination.

- Other co- morbid condition and complications were noted down.

- Relevant laboratory investigations were carried out.

- The following laboratory investigations were carried out as per clinical management protocol.

- $\mathrm{Hb}$

- PCV

- WBC

- Platelets 
- NS1 antigen

- IgM Dengue

- IgG Dengue

- LFT

- RFT

- PT INR

- X-ray chest

- Clinical and consequential data and laboratory interpretation were documented by a predesigned proforma.

- Results were judged, based on the observations, clinical examinations, laboratory investigation and radiological interpretation as per proforma.

- The observations were graphically depicted and with the help of tables and conclusions are drawn on the basis of observations and discussion.

- The data obtained were compiled in tabular form and analysed. Descriptive statistics were used to present data in tabular and graphical forms and suitable statistical tests applied for results.

\section{OBSERVATION AND RESULTS}

1) Distribution of cases according to the diagnosis.

In the current study, maximum patients were found in the DHF group (68 $\%)$ followed by DF (25\%) and DSS (7\%) respectively.

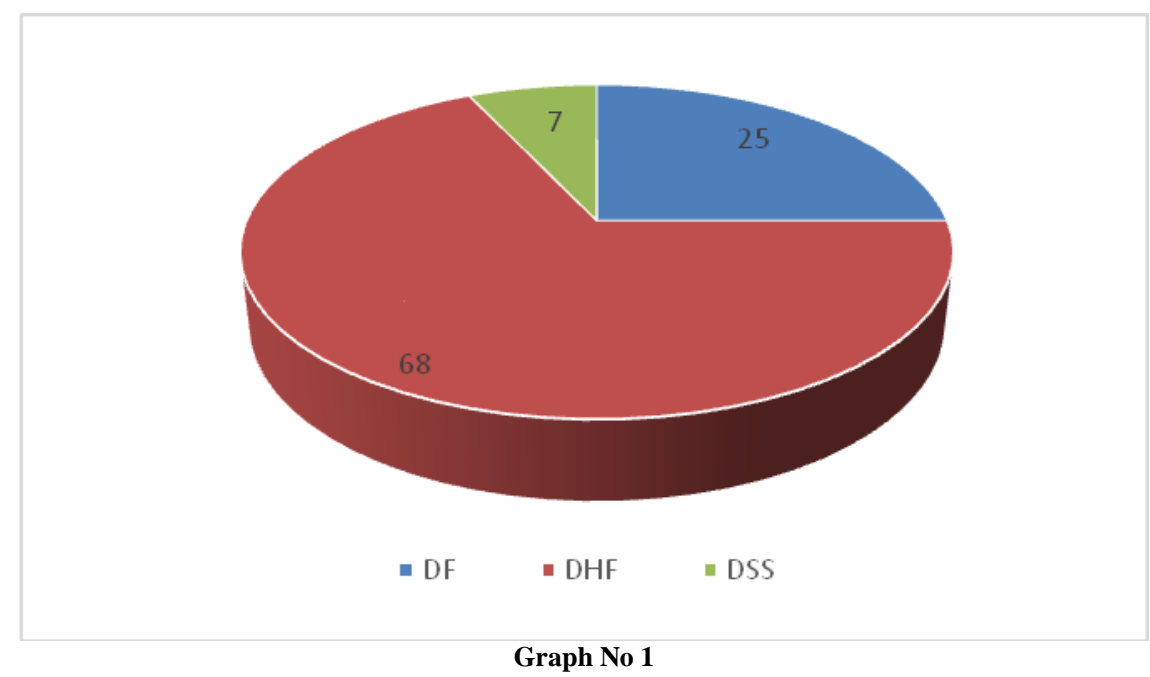

2) Age and gender wise distribution of cases

Maximum patients in the current (46\%), followed by $5-10$ years of age group (29\%). Males are affected more than the females.

study were in the 10 to 15 year age group

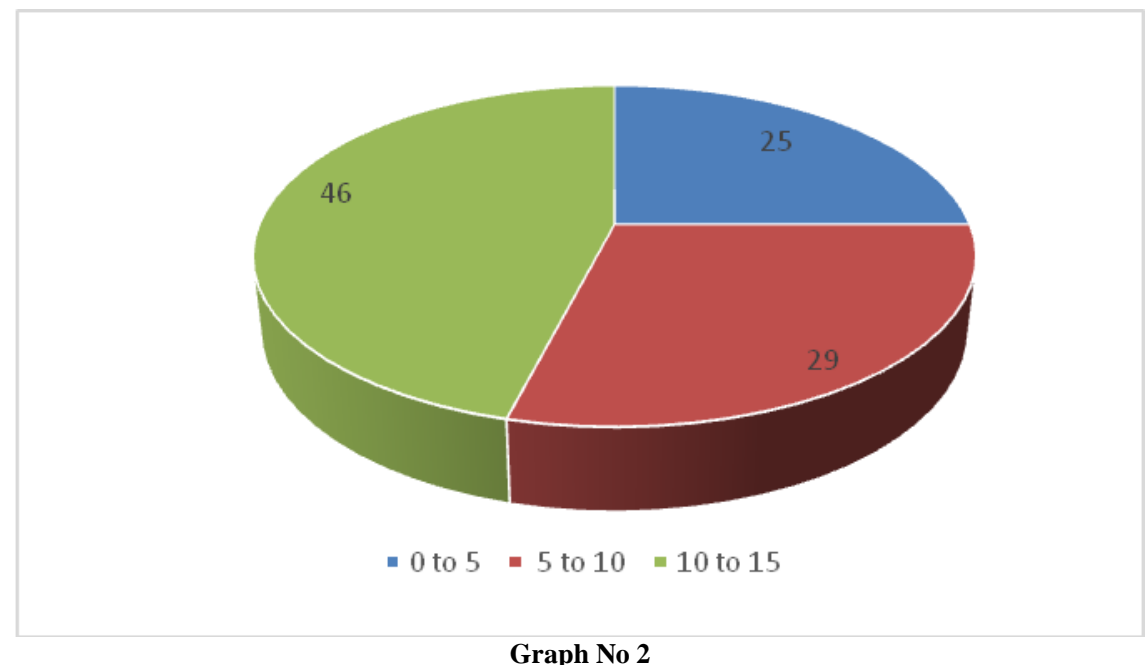




\section{3) Clinical manifestations}

Table no 1:
\begin{tabular}{|l|l|l|}
\hline Clinical manifestations & Number of cases & Percentage \\
\hline Fever & 100 & $100 \%$ \\
\hline Headache & 26 & $26 \%$ \\
\hline Retro orbital pain & 17 & $17 \%$ \\
\hline Arthralgia & 18 & $18 \%$ \\
\hline Body ache & 25 & $25 \%$ \\
\hline Nausea and vomiting & 52 & $52 \%$ \\
\hline Diarrhea & 9 & $9 \%$ \\
\hline Abdominal pain & 25 & $25 \%$ \\
\hline Rash & 54 & $54 \%$ \\
\hline Conjunctival congestion & 13 & $13 \%$ \\
\hline Hemorrhagic manifestations & 58 & $58 \%$ \\
\hline
\end{tabular}

The above table shows that in the current study, fever is the most common clinical feature in dengue $(100 \%)$ followed by Hemorrhagic manifestations (58\%).
4) Elisa NS 1 antigen test, IgM Antibody Test, IgG dengue test

ELISANS 1 antigen test was done in all patients out of which 70 were positive. In 30 patients NS 1 antigen test was negative.

ELISA IgM antibody test was done in all patients, out of which 37 were positive. Both Elisa Ns1 antigen and IgM antibody were positive in 9 patients. $\mathrm{IgG}$ dengue test was positive in 2 patients.

\section{5) Hemoconcentration on admission}

Hemoconcentration was present in $70 \%$ patients on admission

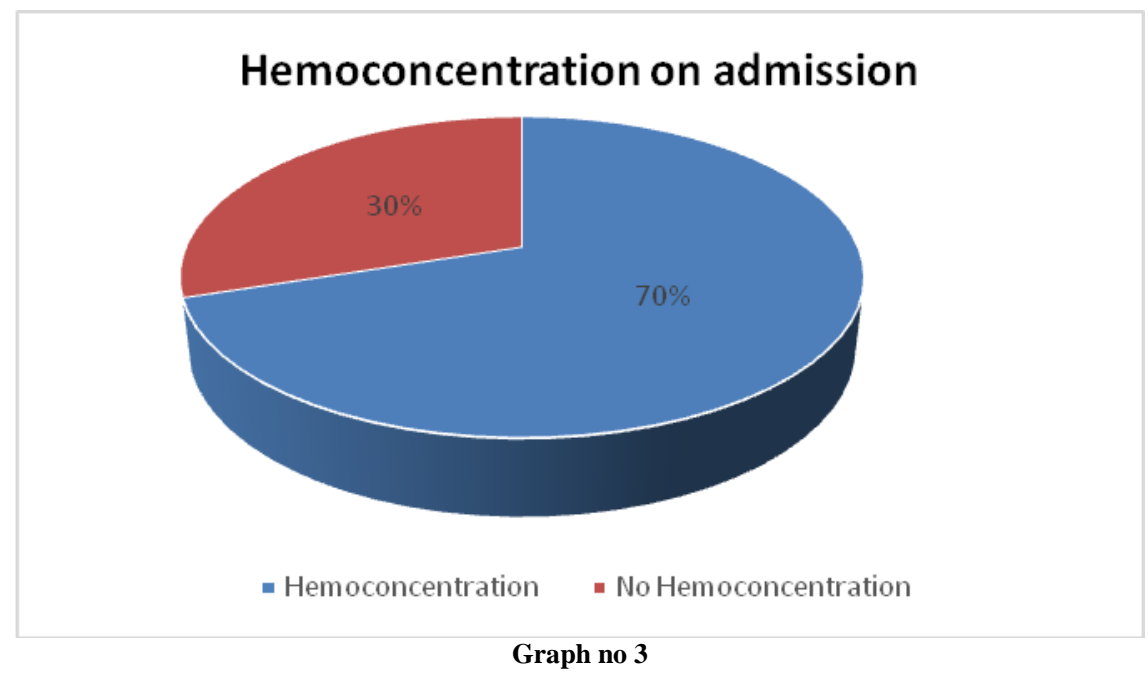

6) On admission, thrombocytopenia:

Table no 3:
\begin{tabular}{|l|l|l|l|l|l|}
\hline Thrombocytopenia (Platelet < 1.5 L/Cu mm & & & & & \\
\hline Yes & N & DF & DHF & DSS & \multicolumn{1}{|c|}{ Total } \\
\hline & $\%$ & 22 & 61 & 7 & 90 \\
\hline No & N & $24.4 \%$ & $67.8 \%$ & $7.8 \%$ & $100.0 \%$ \\
\hline & $\%$ & 3 & 7 & 0 & 10 \\
\hline Total & $\mathrm{N}$ & $30.0 \%$ & $70.0 \%$ & $0.0 \%$ & $100.0 \%$ \\
\hline & $\%$ & 25 & 68 & 7 & 100 \\
\hline & & $100.0 \%$ & $100.0 \%$ & $100.0 \%$ & $100.0 \%$ \\
\hline
\end{tabular}

The above table shows that thrombocytopenia was present in $100 \%$ of the patients.

\section{7) On admission, leukopenia}

Leukopenia was present in $69 \%$ of patients

\section{DISCUSSION}

1) Distribution Of Cases According To Diagnosis
In the current study, $25 \%$ of the patients were of DF, $68 \%$ were of DHF and $7 \%$ were of DSS. In study conducted by Manjith Narayan et al (2000), 33.8\% of patients were of DF, $57.6 \%$ were of DHF and $8.47 \%$ were of $\mathrm{DSS}^{13}$. In the study by Hema Mittal et al (2010), $8 \%$ of the patients were of DF, $51 \%$ were of DHF and $42 \% \mathrm{DSS}^{14}$. In a study conducted by Ashwini Kumar et al (2010), out of total patients admitted, $83.9 \%$ had DF, $8.8 \%$ had 
DHF and $7.3 \%$ had $\mathrm{DSS}^{15}$. We got maximum patients with DHF and DSS as our institute is tertiary referral care centre.

\begin{tabular}{|l|l|l|l|l|}
\multicolumn{5}{|c|}{ Table No 1 } \\
\hline \multirow{2}{*}{ Diagnosis } & $\begin{array}{l}\text { In } \\
\text { current } \\
\text { study }\end{array}$ & $\begin{array}{l}\text { Manjith } \\
\text { Narayan et } \\
\text { al (2000) }\end{array}$ & $\begin{array}{l}\text { Hema } \\
\text { Mittal et } \\
\text { al (2010) }\end{array}$ & $\begin{array}{l}\text { Ashwin } \\
\text { Kumar et } \\
\text { al (2010) }\end{array}$ \\
\hline DF & $25 \%$ & $33.8 \%$ & $8 \%$ & $83.9 \%$ \\
\hline DHF & $68 \%$ & $57.6 \%$ & $51 \%$ & $8.8 \%$ \\
\hline DSS & $7 \%$ & $8.47 \%$ & $42 \%$ & $7.3 \%$ \\
\hline
\end{tabular}

\section{2) Distribution of Cases According to Age Group.}

In the current study, maximum patients were in the 10 to 15 year age group (46\%), followed by 5 to 10 year age group (29\%). In the study by Senthil Kumar K et al(2018), maximum patients were in the more than 10 years age group $(50.7 \%)^{16}$. In a study conducted by Shubhankar Mishra et al(2010) maximum patients were in the age group more than 11 years $(34 \%)^{17}$. This shows that dengue affects school going children more. More prevalence of this age group is probably due to more involvement in outdoor activities during day time exposing them to higher risk of mosquito bites.

\section{3) Male: Female Ratio Among Different Studies}

In the current study, male: female ratio was $2.25: 1$, as compared to $1.32: 1$ in the study by Mittal Hema et al (2010) ${ }^{\mathbf{1 4}}$ and 3.40:1 in the study by Shubhankar et al (2016) ${ }^{17}$. In a study conducted by Ashwini Kumar et al (2010), male: female ratio was $1.83: 1^{15}$.

\section{4) Clinical features among different studies}

In all studies, fever was the most common clinical feature $(100 \%)$. In the current study, the other common features were hemorrhagic manifestations (58\%), $\operatorname{rash}(54 \%)$, nausea/ vomiting (52\%), respiratory symptoms (i.e. cough, cold) $(35 \%)$, bodyache $(25 \%)$, abdominal pain $(22 \%)$.Other manifestations are conjunctival suffusion(13\%) and diarrhoea (9\%).

Fever was invariably high grade with temperature more than $39^{\circ} \mathrm{C}$. Fever in dengue was not responding to normal dose of paracetamol.

Rash was more commonly seen on arms secondary to BP monitoring (Torniquet test). When there is increased bleeding tendency, spontaneous ecchymosis, easy bruising and bleeding at the site of venepuncture were common. Some patients had gross ecchymosis or gastrointestinal bleeding, usually after period of uncorrected shock.

In the study by Ashwin Kumar et al (2010), the common features apart from fever were myalgia $(64.6 \%)$ and vomiting (47.6\%), headache $(47.6 \%)$ and abdominal pain (37.6\%). In the study by Ragini Singh et al (2013), the common features apart from fever were headache $(80 \%)$ and myalgia $(73.6 \%)^{20}$. Rash was present in $15 \%$ cases. In the study by Mittal Hema et al (2002) ${ }^{\mathbf{1 4}}$, bleeding manifestations were found in $48.8 \%$ of the cases. In a study conducted by Senthil Kumar K (2018), fever was present in $93 \%$ patient. Other features were vomiting (68\%), headache $(36 \%)^{16}$.

Table no 2:

\begin{tabular}{|l|l|l|l|l|l|}
\hline Clinical Features & $\begin{array}{l}\text { In } \\
\text { Study }\end{array}$ & $\begin{array}{l}\text { Hema Mittal et al } \\
(\mathbf{2 0 0 2})\end{array}$ & $\begin{array}{l}\text { Ashwini Kumar et } \\
\text { al (2010) }\end{array}$ & $\begin{array}{l}\text { Ragini Singh et al } \\
(\mathbf{2 0 1 3})\end{array}$ & $\begin{array}{l}\text { Senthil kumar K } \\
\text { et al (2018) }\end{array}$ \\
\hline Fever & $100 \%$ & $100 \%$ & $99.1 \%$ & $100 \%$ & $93 \%$ \\
\hline Headache & $26 \%$ & $63 \%$ & $47.6 \%$ & $80 \%$ & $36 \%$ \\
\hline Retroorbital Pain & $17 \%$ & - & - & - & - \\
\hline Arthralgia & $18 \%$ & - & - & - & - \\
\hline Body ache & $25 \%$ & - & $64.6 \%$ & $73.6 \%$ & $11.4 \%$ \\
\hline Nausea and Vomiting & $52 \%$ & - & $47.6 \%$ & $9 \%$ & $68 \%$ \\
\hline Diarrhoea & $9 \%$ & - & $13.9 \%$ & $9 \%$ & $1.2 \%$ \\
\hline Abdominal Pain & $25 \%$ & $71 \%$ & $37.6 \%$ & $13.6 \%$ \\
\hline Rash & $54 \%$ & $26.6 \%$ & $21.7 \%$ & $15 \%$ \\
\hline Hemorrhagic Manifestations & $58 \%$ & $48.8 \%$ & - & $7.1 \%$ \\
\hline Conjunctival Congestion & $13 \%$ & - & - & - \\
\hline
\end{tabular}




\section{5) Elisa NS1 /Igm /IgG}

In the current study, Elisa NS 1 antigen test was done in all patients out of which $68 \%$ were positive. ELISA $\operatorname{IgM}$ antibody Dengue was found to be positive in $30 \%$ patients and $\mathrm{IgG}$ dengue was positive in $2 \%$ patients. Both Elisa NS1 and IgM were positive in $9 \%$ patients. In a study conducted by Senthil Kumar et al (2018), Elisa NS1 antigen assay was positive in $79.1 \%$ patients and Elisa Igm antibody was positive in $14.9 \%$ patients ${ }^{16}$.

In our study, majority of patients were positive for Elisa NS1 followed by Elisa IgM as large number of patients were present within day of illness 5. Elisa Ns1 antigen test helps in early detection of cases.

\section{6) Haematocrit studies}

In the current study, haemoconcentration on admission was present in $63 \%$ of the cases. Haemoconcentration on admission was one of the prominent features in the current study. This was due to the fact that many patients presented late in the course of the illness.

Usually on days 3-7 of illness, an increase in capillary permeability in parallel with increasing haematocrit levels may occur. This marks the beginning of the critical phase. The degree of increase above the baseline hematocrit often reflects the severity of plasma leakage. A hematocrit level increase greater than $20 \%$ is a sign of hemoconcentration and precedes shock. Thus, serial monitoring of haematocrit levels helps in management of patients with dengue.

In the study by Narayan $M$ et al (1998), haemoconcentration was present in $40 \%$ of the cases.

\section{7) Leukocyte counts}

In the current study, leukopenia on admission was present in $69 \%$ of the total dengue patients. There was significant statistical difference among the three groups. In the study by Ch Manoj Kumar et al (2018), leukopenia was present in
$37.5 \%$ of the cases ${ }^{18}$ and it was $41.4 \%$ in the study by Ragini Singh et al (2013) ${ }^{\mathbf{2 0}}$ and $38.9 \%$ in the study by Senthil Kumar K et al (2018) ${ }^{\mathbf{1 6}}$. Thus leukopenia on admission was one of the prominent features in the current study.

\section{8) Platelet counts}

In the current study, thrombocytopenia on admission was present in $90 \%$ of the total dengue patients. In study conducted by Mahesh Ahirrao et al (2019) platelet count was less than 100000 in $51 \%$ patients $^{19}$. In the study by Mittal Hema et al (2002), thrombocytopenia was present in $92.6 \%$ of the cases ${ }^{14}$. In the current study, thrombocytopenia was found more commonly in DHF and DSS.

Platelet counts less than 100,000 cells $/ \mu \mathrm{L}$ are seen in dengue hemorrhagic fever or dengue shock syndrome and occur before defervescence and the onset of shock. Thus, the platelet count monitoring at least every 24 hours helps in early recognition of DHF and DSS.

\section{CONCLUSION}

1) Maximum patients in our study were from 10-15 years of age group, school going children are more exposed to mosquito bites during day time school activities.

2) High grade fever, not responding to routine antipyretics dose should arouse a suspicion of Dengue fever in an epidemic setting.

3) Any fever associated with headache, body ache, erythematous moribiliform rash and conjuctival suffusion should be subjected to Dengue antigen test.

4) ELISA NS1 Ag assay holds promise in early diagnosis of dengue infection. When used in combination with ELISA IgM antibody, it significantly improves the diagnostic algorithm. Early identification of dengue infection using ELISA NS1 antigen test is valuable in terms of disease progression and mortality. 
5) Any patient with symptoms of high grade fever and leukopenia on CBC should be investigated for Dengue fever.

6) Thrombocytopenia is most consistent feature of Dengue hemorrhagic fever and Dengue Shock Syndrome. Many patients with mild dengue fever may have normal platelet count.

\section{ACKNOWLEDGEMENT}

Author would like to thank department faculty and their patients, without their support this study would not have been possible.

\section{Conflict of Interest: None}

\section{Source of Funding: None}

\section{Ethical Approval: Approved}

\section{REFERENCES}

1. https://www.who.int/neglected_diseases/vec tor_ecology/mosquitobornediseases/en/

2. Halstead, Scott B. ((1992. The XXth century dengue pandemic: need for surveillance and research / Scott B. Halstead. World health statistics quarterly $1992 ; 45((3 / 2: 292-298$

3. Jelinek, T., N. Muhlberger, et al. Epidemiology and clinical features of imported dengue fever in Europe: sentinel surveillance data from TropNetEurop." Clinical infectious diseases: an official publication of the Infectious Diseases Society of America. 2002; 35(9): 10471052.

4. Bhatt, S., et al., The global distribution and burden of dengue. Nature, 2013. 496(7446): p. 504-507.

5. A global brief on vector borne disease apps. who.int > bitstream >111008: 15

6. Global strategy for dengue prevention and control. 9789241504034_eng: 6

7. Hanley KA, Weaver SC. Arbovirus Evolution. In: Domingo E, Parrish CR, Holland JJ, editors. Origin and Evolution of Viruses. Elsevier; Oxford: 2008: 351-392.

8. Westaway E G, Blok J. Taxonomy and evolutionary relationships of f7laviviruses. In: Gubler D J, Kuno G, editors. Dengue and dengue hemorrhagic fever. London,
United Kingdom: CAB International; 1997:147-173.

9. https://www.who.int/denguecontrol/mosquit o/en/

10. WHO SEARO. Comprehensive Guidelines for Prevention and Control of Dengue and Dengue Haemorrhagic Fever Revised and expanded 2011.

11. Park K. "The Dengue Syndrome" In Park's Textbook of Preventive and Social Medicine 21st Edition. 5(3): 224-231.

12. Dr Deen Jacqueline, Dr Lum Lucy, Dr Martinez Eric, Dr Tan Lian Huat. "Clinical management and delivery of clinical services." WHO Dengue guidelines for diagnosis, treatment, prevention and control, New edition 2009. 2: 25-54.

13. Manjith Narayanan, M.A. Aravind, N. Thilothammal, R. Prema, C.S. Rex Sargunam and Nalini Ramamurty. Dengue Fever Epidemic in Chennai - A Study of Clinical Profile and Outcome. Indian Pediatrics 2002; 39:1027-1033.

14. Hema Mittal, M. M. A. Faridi, Shilpa Khanna Arora, Rahul Patil. Clinicohematological Profile and Platelet Trends in Children with Dengue during 2010 Epidemic in North India. The Indian Journal of Pediatrics 2012 April; 79(4): 467-471.

15. Ashwini Kumar, Chythra R Rao, Vinay Pandit, Seema Shetty, Chanaveerappa Bammigatti, and Charmaine Minoli Samarasinghe. Clinical Manifestations and Trend of Dengue Cases Admitted in a Tertiary Care Hospital, Udupi District, Karnataka. Indian J Community Med. 2010 July; 35(3): 386-390.

16. Senthil Kumar K., Rajendran N. K, Ajith Brabhukumar C. Clinical profile of dengue fever in children: analysis of 2017 outbreak from Central Kerala, India. International Journal of Contemporary Pediatrics 2018 Nov; 5(6): 2265-2269.

17. Shubhankar Mishra, Ramya Ramanathan, and Sunil Kumar Agarwalla. Clinical Profile of Dengue Fever in Children: A Study from Southern Odisha, India. Scientifica 2016; Volume 2016

18. Ch. Manoj Kumar, K. S. Keerthi Vyas, Y. Sai Krishna.Clinical profile of dengue fever with severe thrombocytopenia and its complications: a retrospective study at a tertiary care hospital in South India. 
19. Mahesh H Ahirrao, Bhagyashri M Ahirrao, Nandkumar V Dravid, Aashish D Shah. Evaluation of Rapid Immunochromatographic Tests in Comparison to ELISA for Detection of NS1, IgM and IgG Antibodies for Early Detection of Dengue Infection in Pediatric Patients2019;5(4):1-6

20. Ragini Singh, S. P. Singh, Niaz Ahmad. A study of clinical and laboratory profile of dengue fever in a tertiary care centre of
Uttarakhand, India. International Journal of Research in Medical Sciences 2014 Feb; 2(1):160-163.

How to cite this article: Ahirrao M, Patil A, Kurup A et.al. Clinical profile and laboratory findings of dengue fever in children. Int $J$ Health Sci Res. 2021; 11(5):30-37. DOI: https://doi.org/10.52403/ijhsr.20210504 